\title{
Future passenger mobility
}

\author{
Embedding behavioural, environmental and health considerations, alongside economic needs, into transport \\ policies can pave the way to a sustainable system.
}

T he demand for electric vehicles (EV) is growing worldwide. According to the Global EV Outlook 2019 report by the International Energy Agency, electric car use has grown rapidly over the past ten years, with the global stock of electric passenger cars at above 5 million in 2018 an increase of $63 \%$ from 2017. The largest proportion of electric cars on the road in 2018 was found in China ( $45 \%$ of the global fleet), followed by Europe (24\%) and the United States (22\%). Yet things are changing rapidly. Late in May, it was reported that Europe has recently seen a sharp increase in financial investment to step up EV production, surpassing China, most probably as a result of the stringent $\mathrm{CO}_{2}$ emissions targets set by the European Union (EU). Indeed, given the most recent $\mathrm{EU}$ official target, manufacturers are required to reduce their fleet-wide carbon footprint to an average of $95 \mathrm{~g} \mathrm{~km}^{-1}$ by 2021 or otherwise they could face fines for billions of euros. Whilst projected EV sales suggest continued growth throughout 2021 with a strong market performance in Europe, there are expectations of positive growth also in the US market as more car models roll out.

Despite the recent electric car market push in the face of tight climate change targets, concerns about the actual climate benefits of electrifying passenger transport remain. It is unclear to what extent overall carbon emissions would decrease as a result of replacing conventional cars with electric ones, when electricity production is not yet fully decarbonized. In an Article in this issue, Knobloch and colleagues examine the effects of electrifying passenger cars and household heating systems under different timings of decarbonization of electricity generation. The analysis covers 59 world regions and draws from a thorough life-cycle assessment of all relevant production-related and use-related emissions of the current technologies in all their aspects, now and in the future. The researchers look at things like which type of fossil-based car is going to be replaced by which type of electric car (reflecting heterogeneous individual behaviour), rather than simply comparing generic technology types. And they look at when replacement might take place, considering different stages

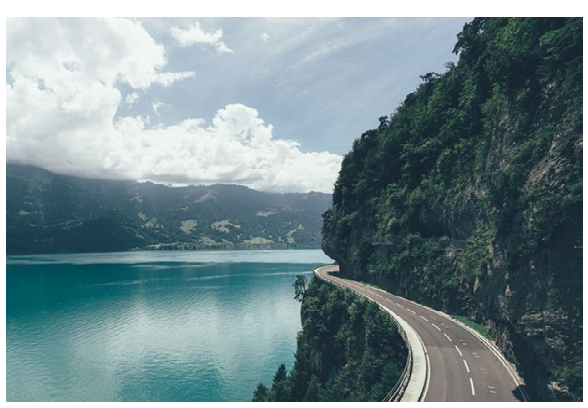

Credit: Ueli Frischknecht / EyeEm / EyeEm / Getty

of decarbonization of the power system. Overall, they find that even if end-use electrification - replacing conventional cars with electric ones and fossil-based boilers with heat pumps - is not matched by rapid power-sector decarbonization, it will most likely reduce carbon emissions in almost all world regions.

Governments should support electric mobility as part of climate change mitigation strategies. But what about EV users' experiences? Is the general public confident about the reliability of the existing charging infrastructure for electric cars? Are people having different experiences when using charging stations in publicly owned locations versus privately owned ones? In an Analysis in this issue, Asensio and co-authors provide answers to those questions in the context of the United States. They use machine-learning algorithms to automatically classify more than 120,000 consumer reviews provided by a popular EV charge station locator app at 12,720 EV charging stations across the United States. The classification allows the researchers to extract valuable behavioural insights about EV users' experiences and perceptions. They find that about half of the drivers who use mobility apps have had negative experiences at EV charging stations in the early growth years of public charging infrastructure. However, and contrary to expectations, charging stations at privately owned locations do not outperform publicly owned ones. Overall the study suggests that, as EV infrastructure grows it is not only the quantity of available stations that matters to consumers, but also the quality of the charging experience. Policies should increasingly take behavioural insights into account.

Besides climate change, there are also other reasons why electrifying transport would be socially beneficial, for example ambient air pollution and public health. In an Article published last year, $\mathrm{Wu}$ and co-workers showed that in China fleet electrification can synergistically deliver greater air quality, climate change and public health benefits. However, a full electrification of transport is unlikely to happen overnight. For some time, conventional vehicles and electric ones will coexist. Hence, while policies to push electrification are tightened, it remains critical to find ways to reduce energy and emission intensity of conventional vehicles. In another Analysis, Schäfer and Yeh in this issue examine key indicators of energy use and emissions from transport in different regions around the world. More specifically, they look at the energy, and carbon emission, intensity of transport modes including light-duty vehicles, buses, railways and fixed-wing aircraft. They define energy, and emission, intensity as energy use, or greenhouse gas (GHG) emissions, per passenger-kilometres travelled. They find that even for individual modes, differences in vehicle occupancy can cause changes in energy and GHG intensity of up to one order of magnitude. The researchers conclude that vehicle occupancy per se could be used to carry out first-order estimates of a transport mode's energy intensity and therefore highlight the need of transport policy interventions aimed at increasing vehicle occupancy to curb carbon emissions.

Mobility is crucial to the functioning of societies and economies around the world but governments should not neglect the environmental and public health implications of different transport solutions, nor should they ignore consumers' sentiments. It's only by designing fully integrated interventions that they can ensure a transition to a more sustainable transport system.

Published online: 16 June 2020

https://doi.org/10.1038/s41893-020-0564-z 